\title{
Tuning capillary penetration in porous media: combining geometrical
}

\section{and evaporation effects}

\author{
Mingchao Liu, ${ }^{\dagger}$ Jian $\mathrm{Wu},{ }^{\S}$ Yixiang Gan, ${ }^{\ddagger}$ Dorian AH Hanaor, ${ }^{\sharp}$ and C.Q. Chen ${ }^{\dagger, 1}$ \\ †Department of En gineering Mechanics, CNMM \& AML and ${ }^{\S}$ AML \& Center for Flexible Electronics Technology, \\ Department of Engineering Mechanics, Tsinghua University, Beijing 100084, China \\ tSchool of Civil Engineering, The University of Sydney, Sydney, NSW 2006, Australia \\ "Institute for Materials Science and Technology, Technische Universität Berlin, Berlin 10623, Germany
}

\section{Graphical abstract}
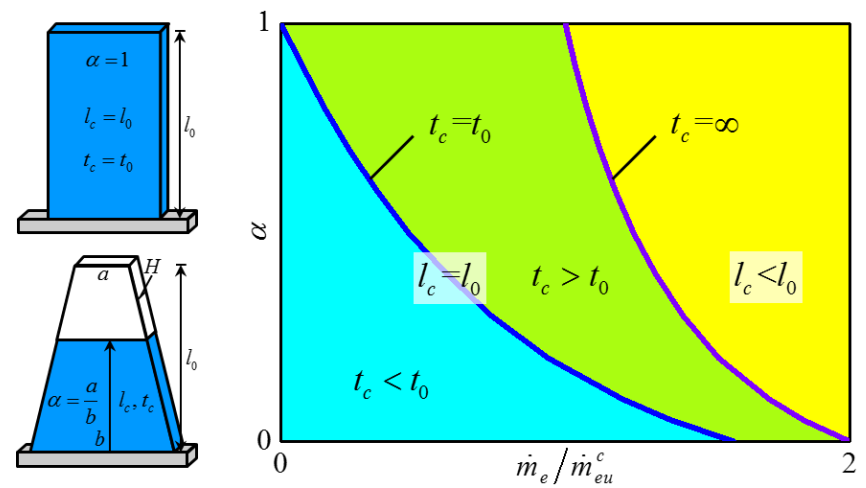

\begin{abstract}
Capillary penetration of liquids in porous media is of great importance in many applications and the ability to tune such penetration processes is increasingly sought after. In general, liquid penetration can be retarded or restricted by the evaporation of volatile liquid at the surface of the porous media. Moreover, when capillary penetration occurs in a porous layer with non-uniform cross section, the penetration process can be accelerated or impeded by adjusting the section geometry. In this work, on the basis of Darcy's Law and mass conservation, a theoretical model of capillary penetration combining evaporation effects in two-dimensional homogeneous porous media of varying cross-section is developed and further examined by numerical simulations. The effects of sample geometry and liquid evaporation on capillary penetration are quantitatively analyzed. Results show that the penetration velocity is sensitive to the geometry of the porous layer, and can be tuned by varying the evaporation rate for a given geometry. Under given evaporation conditions, penetration is restricted to a limited region with a predictable boundary. Furthermore, we find that the inhibition of liquid penetration by evaporation can be offset by varying the geometry of the porous layer. In addition, the theoretical model is further extended to model the capillary flow in three-dimensional porous media, and the interplay of geometry and evaporation during the capillary flow process in $3 \mathrm{D}$ conditions is also investigated. The results obtained can be used for facilitating the design of porous structures, achieving tunable capillary penetration for practical applications in various fields.
\end{abstract}

Keywords: Porous media; Tunable capillary penetration; Geometrical shape; Evaporation effect

\footnotetext{
${ }^{1}$ Corresponding author. Tel/Fax: +86 10 62783488; Email: chencq@ @singhua.edu.cn (C.Q. Chen)
} 
1.

\section{Introduction}

When a dry porous medium contacts a liquid reservoir, the liquid is transported into the porous medium driven by capillary force. This phenomenon is known as capillary penetration. Capillary penetration in porous media is commonly observed in phenomena such as water absorbing into paper [1] and rising damp in concrete walls [2]. Recently, capillary penetration has attracted increasing scientific and industrial attention, owing to the high value of its diverse contemporary applications including paper-based microfluidics [3, 4], medical diagnosis [5], energy-harvesting devices [6,7], advanced textile engineering [8,9], cooling devices [10], architectural conservation [11], and oil recovery [12]. Capillary penetration has also been utilized as an inverse method to determine the effective properties (e.g., the pore size distribution and porosity) of porous media in both numerical $[13,14]$ and experimental [15-17] approaches.

Capillary penetration in porous media shares a similar dynamic mechanism with capillary flow in hollow tubes, with both processes resisted by viscous forces [18]. For a capillary tube with a one-dimensional (1D) uniform geometry, dynamic liquid penetration is quantified by a diffusive relationship between the position of the liquid front $L$ and time $t$, i.e., $L^{2}=D t$, where $D$ is the diffusive coefficient depending on the tube size and the liquid properties [19]. This relationship is best known as the "Lucas-Washburn (LW) law", presented by Lucas [20] and Washburn [21] a century ago. This classical result has been found to be valid for both unidirectional and radial capillary penetration in porous media [22-25], and is further extended to the hemispherical penetration in a semi-infinite porous medium [26] and imbibition in structured porous media with axially variable geometries [27]. However, recent studies have also indicated that this simple model is not applicable for some complex cases, such as flow processes in heterogeneous and random porous media, and some other effects, e.g., fractal and disorder, should be incorporated into the analysis [28-32].

Tuning capillary penetration processes in porous media, with an emphasis on penetration velocity, is of increasing interest in the burgeoning field of microfluidics [33]. However, the control of fluid flow is not readily achievable by tuning the pore size and porosity for most commonly used porous media such as paper substrates [34]. For the purpose of facilitating control, applied porous media are commonly considered to have different cross sections in the direction of capillary flows [27, 35]. Recently, Benner and Petsev [36] pointed out that varying the shape of a porous material leads to qualitative differences in the resulting flow patterns. Additionally, Shou et al. [37] explored the geometry-induced asymmetric capillary flow in porous structures. They found that the geometrical shape has a significant effect on the flow behavior. Subsequently, rational design of porous structures for enhanced and controlled capillary flows have been investigated [38, 39]. Notably, previous studies have revealed that the capillary penetration in nonuniform porous structures should be treated as a two- (2D) or three-dimensional (3D) situation, and the time dependence of the flow deviates from the LW 1D case [26, 36, 39].

A key factor affecting capillary penetration is 
the evaporation of liquid from the surface of porous media [40, 41], with particular relevance for highly volatile liquids, such as the detecting reagent, used in paper-based sensors and diagnostics [42]. Evaporation also has a significant effect on some practical respects, such as soil penetration of water in irrigation processes [43], liquid flow in fuel cells [44], and liquid transfer in capillary evaporators [45]. Experimental results revealed that capillary penetration in porous media is strongly affected by evaporation, which restricts liquid penetration to a limited region [46]. In order to quantitatively investigate the evaporation effect in unidirectional penetration, the Lucas-Washburn law was employed and augmented by Fries et al. [40] to model dynamic wicking processes. Recently, Liu et al. [47] reported a model based on Darcy's law and the principle of mass conservation for radial capillary penetration, in which the boundary of the limited liquid penetrated region was predicted theoretically. In their study the geometry was held constant and thus the penetration process was not tunable.

Although capillary penetration in porous systems has been extensively investigated, little work has dealt with the combined effects of geometry and evaporation during capillary penetration. The development of capillary-driven microsystems for energy and biotechnology applications requires precise control and regulation of the penetration process, including velocity and total penetration time. An improved mechanistic understanding of capillary penetration with combined geometrical and evaporation effects will facilitate the application and design of porous structures. From this perspective, we present here a theoretical and numerical study on the combined effects of geometry and evaporation on penetration processes in porous media, with a view towards tunable capillary flow. Porous structures with 2D non-uniform cross-sectional geometric shape are considered first, and the analysis is further extended to $3 \mathrm{D}$ porous structures.

\section{Theoretical model}

To investigate the combined effects of geometry and evaporation on the capillary penetration through homogenous porous media, a trapezoidal thin porous plate with one end contacting with an unlimited reservoir is considered, as shown in Fig. 1. As a simple case of $2 \mathrm{D}$ porous sample with a non-uniform cross section, the trapezoidal structure has essential geometric features such as asymmetry and shape variation that facilitate the investigation of the basic effect of the sample geometry on fluid penetration in porous media [37]. The penetration processes takes place from the liquid reservoir to the other end of the plate (see, Fig. 1a), and is weakened by the concurrent evaporation of liquid from the plate surfaces (see Fig. 1b). 

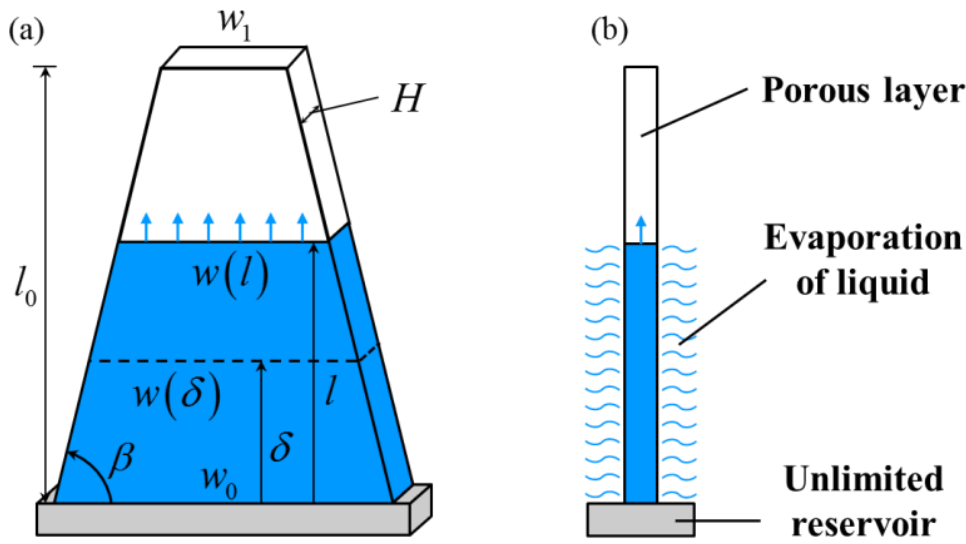

FIG. 1. Schematic illustration of capillary penetration incorporating evaporation effect in a trapezoidal porous plate: (a) oblique view; (b) side view.

As shown in Figure 1a, the considered porous plate has a trapezoidal geometrical shape. The width of the plate varies with the position as the following relation,

$$
w(\delta)=w_{0}\left[1-\frac{\delta}{l_{0}}(1-\alpha)\right],
$$

where $w_{0}$ is the width of the edge in contact

with the reservoir, $l_{0}$ the length of the plate sample, and $\delta$ the local position in the penetrated region. The thickness of the porous plate $H$ is much smaller than its length dimension $l_{0}$ such that it can be treated as a planar problem. To study the effect of plate geometry we define a parameter $\alpha=w_{1} / w_{0}$, where $w_{1}$ is the width of the top edge. The geometry of the plate can also be characterized by the base angle $\beta$ of the trapezoid, which is related to the geometrical factors by

$$
\cot \beta=\frac{w_{0}}{2 l_{0}} \cdot\left\{\begin{array}{l}
(\alpha-1), \alpha \geq 1 \\
(1-\alpha), \alpha<1
\end{array}\right. \text {. }
$$

It is noteworthy that the liquid penetration in this trapezoidal porous plate is technically not a simple 1D problem. As discussed in the previous work [36], the liquid flux in the transverse direction of the flow is not generally zero and depends on the opening angle of the expansion (contraction), which is different from the penetration in a uniform plate (i.e., $\alpha=1$ ). The liquid front is in general not a straight line perpendicular to the flow direction, which is involved in the 1D case, but an elliptic curve. Therefore, this case should be regarded as a 2D problem. According to the results of Elizalde et al., [39] however, the relative error of flat liquid front assumption in the 1D model, in comparison with the 2D model, is small even if the opening angle as large as $90^{\circ}$, which corresponds to a base angle $\beta$ of $135^{\circ}$ in our model. Therefore, to simplify the analysis, we adopt the 1D assumption of flat liquid front (see Fig. 1a) to establish the theoretical model involving the evaporation effect, and the predictions are further examined using numerical simulations in following Section.

We consider the evaporation of liquid from the penetrated region shown in Fig. 1b. A 
constant and uniform evaporation rate is assumed for a given liquid, ambient temperature, and relative humidity. The total mass flow rate due to evaporation can be calculated as

$$
\dot{M}_{e}=2 \cdot \dot{m}_{e} \cdot \frac{1}{2}[w(0)+w(l)] \cdot l,
$$

where $\dot{m}_{e}$ is the evaporation rate, which is used to characterize the evaporation of liquid (i.e., the mass loss due to the evaporation per area and time) with the dimension of $\left[\mathrm{kg} / \mathrm{m}^{2} \cdot \mathrm{s}\right]$, and $l$ is the length of the penetrated region. From Eq. (1), $w(0)$ can be calculated as $w_{0}$. Note that the factor "2" in the right side of Eq. (3) indicates the assumption of equal evaporation from front and back surfaces in Fig. 1a. Moreover, evaporation from the secondary side surfaces are neglected owing to the negligible thickness assumed here.

When evaporation is included, mass transfer in porous media conforms to the law of mass conservation. For the considered thin porous plate with porosity $\phi$ and assuming negligible volume changes, the conservation of mass can be expressed as

$\dot{\delta} \cdot w(\delta) H \phi=i \cdot w(l) H \phi+2 \cdot \frac{\dot{m}_{e}}{\rho} \cdot \frac{1}{2}[w(\delta)+w(l)] \cdot(l-\delta)$

where $\rho$ is the fluid density, and $\dot{\delta}=d \delta / d t$ and $i=d l / d t$ are the local fluid velocity and the liquid front velocity, respectively. Furthermore, in the penetration process, the flow rate $Q$ in a porous medium is obtained based on Darcy's law as

$$
Q=\dot{\delta} A \phi=-\frac{A k}{\mu} \frac{\partial P}{\partial \delta},
$$

where $A=w(\delta) \cdot H$ is the cross sectional area of the plate at position $z, k$ is the permeability of the porous medium, $\mu$ is the viscosity of the liquid, and $P$ is the pressure of liquid.

Combining Eqs. (4) and (5), one obtains

$$
\begin{aligned}
P_{c} & =\frac{\phi \mu}{k}\left(l+\frac{l_{0}}{\alpha-1}\right) \ln \left(1+\frac{l}{l_{0}}(\alpha-1)\right) \frac{d l}{d t} \\
& +\frac{\mu \dot{m}_{e}}{k \rho H}\left[\left(l+\frac{l_{0}}{\alpha-1}\right)^{2} \ln \left(1+\frac{l}{l_{0}}(\alpha-1)\right)-\frac{l}{2}\left(l+\frac{2 l_{0}}{\alpha-1}\right)\right]
\end{aligned}
$$

where $P_{c}=2 \sigma \cos \theta_{s} / R_{\text {eff }}$ is the capillary force, governed by the air-liquid surface tension, $\sigma$, the equilibrium contact angle of the liquid with the solid, $\theta_{s}$, and the effective pore radius of the porous medium, $R_{\text {eff }}$. The second part of the right side in Eq. (6) refers to the evaporation-induced viscous pressure loss $P_{m}$, which represents the viscous resistance to liquid front movement in the penetration process. According to the above equation, the penetration distance (i.e., the liquid front position) can be predicted as a function of time. It should be noted that this model is valid only for cases exhibiting a sharp liquid front, i.e., the penetrated region behind the interface is fully-saturated. In addition, its validity is limited to horizontal penetration or vertical penetration without gravity. 
Asymmetric penetration is examined by changing the value of $\alpha$. As a limiting case of uniform cross section, $\alpha=1$, Eq. (6) reduces to the solution provided by Fries et al. $^{40}$ Another limiting case is that of negligible evaporation, $\dot{m}_{e}=0$, whereby Eq.

(6) reduces to the classical model [38].When $\alpha=1$ and $\dot{m}_{e}=0$ both meet, the original

LW relation $[20,21]$ is recovered.

\section{Numerical simulation and results analysis}

\subsection{Numerical methods}

As mentioned in the preceding section, a flat liquid front is assumed in the theoretical model, which is only accurate and valid for the 1D situation. COMSOL Multiphysics 5.2a finite element software (COMSOL Inc., Burlington, MA) is used to simulate a full 2D problem to verify the proposed model (i.e., eq. (6)).

The 2D capillary penetration process in porous media can be controlled by a set of simultaneous partial differential equations, viz., Darcy's law

$$
\mathbf{v}=-\frac{k_{i}}{\mu} \nabla P
$$

and the conservation of mass

$$
\nabla\left[\rho\left(-\frac{k_{i}}{\mu} \nabla P\right)\right]=F \text {, }
$$

where $\rho$ is the fluid density, $k_{i}=k / \phi$ is the interstitial permeability, and $F$ is a source term, which is related to the evaporation of liquid. For negligible evaporation, $F$ is set to zero; otherwise, it can be calculated as $F=\dot{m}_{e} / H \phi$.

The above Eqs. (7) and (8) govern the 2D penetration process in the full region. When the cross section of the region is uniform, they reduce to the 1D case, as given by Eqs. (5) and (4) with $\alpha=1$. By solving the set of equations, we can obtain the distribution of liquid flow velocity and the boundary of the penetration region at any time.

In finite element analysis, the boundary conditions of the numerical model are defined such that the left and right surfaces are non-penetrable and symmetric and the reservoir-contacting boundary is stationary. Note that the reservoir is exposed the ambient atmosphere and thus the atmospheric pressure, $P_{\text {atm }}$, is fixed at the reservoir-contacting edge, while the pressure at the fluid front is set as $P_{\text {atm }}-P_{c}$, where $P_{c}$ is the capillary pressure. To track the velocity of a moving liquid front, a Moving Mesh module is used in the COMSOL software [35]. To accommodate upward motion of the boundary of the fluid front, side boundaries are allowed to be stretched in the direction of flow, but not in the direction perpendicular to flow. As the liquid front moves automatic re-meshing is implemented to avoid mesh distortion. Moreover, a mesh sensitivity study has also been conducted a priori to ensure the convergence of the numerical models.

\subsection{Results and analysis}

By applying the developed numerical model, the capillary penetration process can be simulated for different geometrical shapes and evaporation conditions. The simulation 
results of the time dependent penetration distance are shown in Fig. 2 as symbols. Also included are the theoretical predictions given by Eq. (6), represented by lines. Two sets of geometrical shapes, (a) $\alpha=100 \quad$ (with three base angles $\beta=105^{\circ}, 120^{\circ}$, and $135^{\circ}$ )

and (b) $\alpha=0.01$ (with $\beta=45^{\circ}, 60^{\circ}$, and $\left.75^{\circ}\right)$, are considered under different evaporation rates (i.e., $\dot{m}_{e} / \dot{m}_{e}^{c}=0,1,2$ and

5). The results are made dimensionless by two scaling parameters $l_{0}$ and $t_{0}$, where $l_{0}$ is the length of the plate sample, and

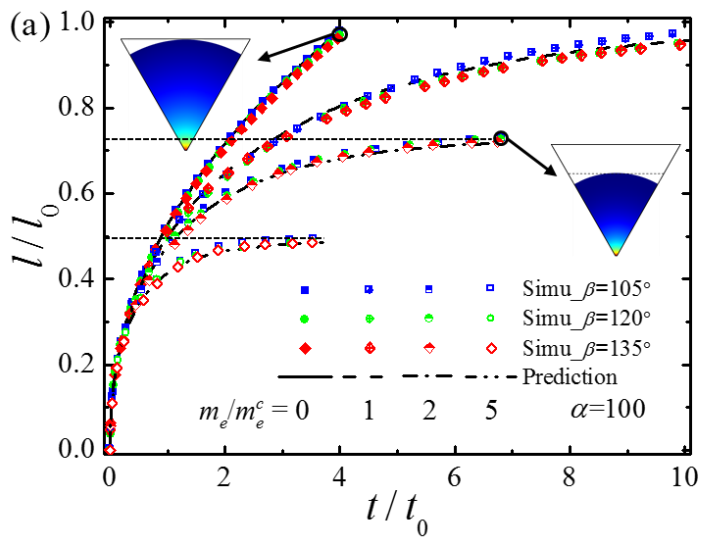

$t_{0}=\mu \phi l_{0}^{2} / 2 k P_{c}$ is the time scale of the liquid penetrating from the reservoir to the other end of a uniform porous plate (i.e., the special case of $\alpha=1$ ). The parameter $\dot{m}_{e}^{c}$ is the critical evaporation rate that allows liquid to reach the end of the porous plate away from the reservoir. The definition and characteristics of $\dot{m}_{e}^{c}$ will be discussed later. For illustration, the representative simulated results of penetration region with pressure profiles (i.e., (a) $\beta=120^{\circ}$ and (b) $\beta=$ $60^{\circ}$ with $\dot{m}_{e} / \dot{m}_{e}^{c}=0$ and 2) are included as inserts in Fig. 2.

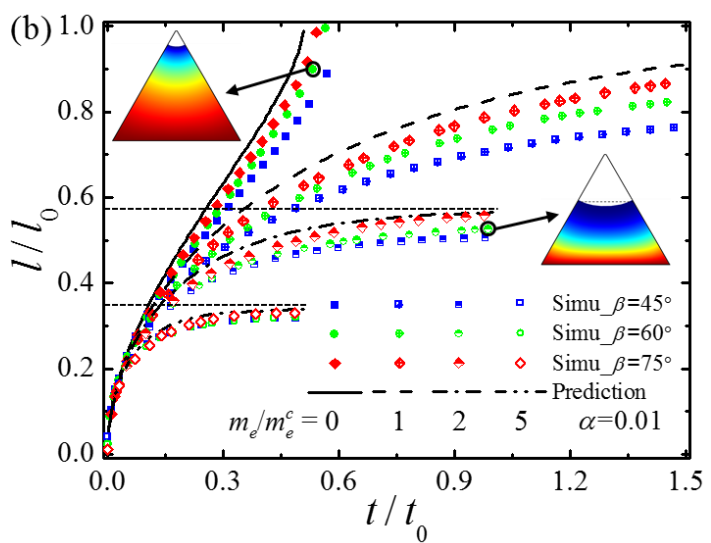

FIG. 2. Comparison of numerical simulations (symbols) and theoretical predictions (lines) of penetration distance versus time for asymmetric capillary penetration in a porous sample with (a) $\alpha=100$ (with base angles $\beta=105^{\circ}, 120^{\circ}$, and $\left.135^{\circ}\right)$ and (b) $\alpha=0.01\left(\beta=45^{\circ}, 60^{\circ}\right.$, and $\left.75^{\circ}\right)$ under different evaporation rates $\left(\dot{m}_{e} / \dot{m}_{e}^{c}=0,1,2\right.$ and 5). Dotted lines indicate the critical penetration distance. The insets show the simulated results of the penetration region with pressure profiles. The contours of the insets, from red to blue, indicate the pressure level varying from $P_{\text {atm }}$ to $P_{\text {atm }}-P_{c}$.

It can be clearly seen from the inserts in Fig. 2 that the liquid fronts are not straight lines in the simulations. For $\alpha=100$, the liquid front is a convex curve (relative to the reservoir position), but a concave curve for $\alpha=0.01$. For simplification, we choose the middle point of the concave/convex curve to represent the liquid front position, with results are plotted in Fig. 2 as symbols. It should be noted that other selections of the representative point for the liquid front position are also feasible. Although those selections may lead to different relative errors, they do not affect the conclusion 
Cite as: Liu, M., Wu, J., Gan, Y., Hanaor, D. A., \& Chen, C. Q. (2018). Tuning capillary penetration in porous media: combining geometrical and evaporation effects. International Journal of Heat and Mass Transfer, 123, 239-250.

drawn here. Numerical simulation and theoretical predictions match closely for all geometrical shapes and evaporation conditions. Specifically, relative error for all the three cases of $\alpha=100$ (i.e., $\beta=$ $105^{\circ}, 120^{\circ}$, and $135^{\circ}$ ) is less than $5 \%$. The small error found may arise owing to the liquid flow velocity, which depends on the pressure gradient following Darcy's law, and is almost the same at the centre line in 1D and 2D conditions. However, for the set of $\alpha=0.01$ (i.e., $\beta=45^{\circ}, 60^{\circ}$, and $75^{\circ}$ ), due to the effect of the initial reservoircontacted boundary condition being more significant than for the case of $\alpha=100$, the relative error is also greater. It can be found that the relative error is larger for smaller base angles and larger evaporation rates, and the maximum value at the extreme case of $\beta=45^{\circ}$ and $\dot{m}_{e} / \dot{m}_{e}^{c}=0$ is about $20 \%$.

While for the cases of $\beta=60^{\circ}$ and $75^{\circ}$ at the same condition, relative errors are approximately $10 \%$ and 5\%, respectively. Consequently, the theoretical model based on a 1D assumption is considered valid for most samples with non-uniform geometrical shape.

Figure 2 also shows the evaporation effect on asymmetric capillary penetration. It can be seen that the penetration distance $l / l_{0}$ increases with time $t / t_{0}$ and the speed of penetration is smaller for larger values of $\dot{m}_{e} / \dot{m}_{e}^{c}$ for both two sets of geometries ( $\alpha=100$ and $\alpha=0.01$ ). In similarity to the case of radial capillary penetration, ${ }^{47}$ we identify a critical state whereby the penetrating liquid can just reach the end of the porous plate under conditions of $\dot{m}_{e}=\dot{m}_{e}^{c}$, as shown by the dash lines in Fig. 2. For evaporation rates greater than the critical value, i.e., $\dot{m}_{e} / \dot{m}_{e}^{c}>1$, the penetration distance approaches an asymptote (i.e., the critical $l / l_{0}$ ) for sufficiently long timeframes. It is thus an evaporation-limited penetration, with the critical length of penetration decreasing with increasing the evaporation rate. In contrast, when $\dot{m}_{e} / \dot{m}_{e}^{c} \leq 1$, liquid penetrates into the entire region for all geometries.

For unlimited penetration, under conditions of $\dot{m}_{e} / \dot{m}_{e}^{c}<1$, it is interesting to note that the total penetration time also depends on the geometrical shape for a given evaporation rate. Specifically, penetration is faster for samples with small values of $\alpha$. Moreover, the penetration velocity for the case of $\alpha=100$ (see Figure 2a) decreases over the entire penetration process. However, for the case of $\alpha=0.01$ shown in Figure 2b, it is obvious that the penetration velocity first increases and then decreases with increasing time. This indicates that we can tune the capillary penetration process, e.g., the penetration velocity or the total penetration time, by controlling the geometrical shape of the porous layer and the evaporation rate of the liquid.

\section{Tuning capillary penetration in porous media}

With the sustained development of capillarybased microsystems, such as paper-based microfluidics, medical diagnosis and energyharvesting devices [3-7], precisely tuning of the capillary penetration process in porous 
media is of emerging interest for both scientists and engineers in recent years.

\subsection{Transition of the penetration velocity}

Capillary-driven devices for energy and biotechnology applications require precise control of the capillary penetration velocity. As shown in the preceding section, for some porous samples with special geometrical shapes under the appropriate evaporation conditions, the penetration velocity can increase with time following an initial deceleration. This interesting feature of tunable capillary penetration has ramifications towards potential multifunctional applications in microfluidic devices for chemical analysis and catalysis, which necessitate the precise control of flow velocity [48].

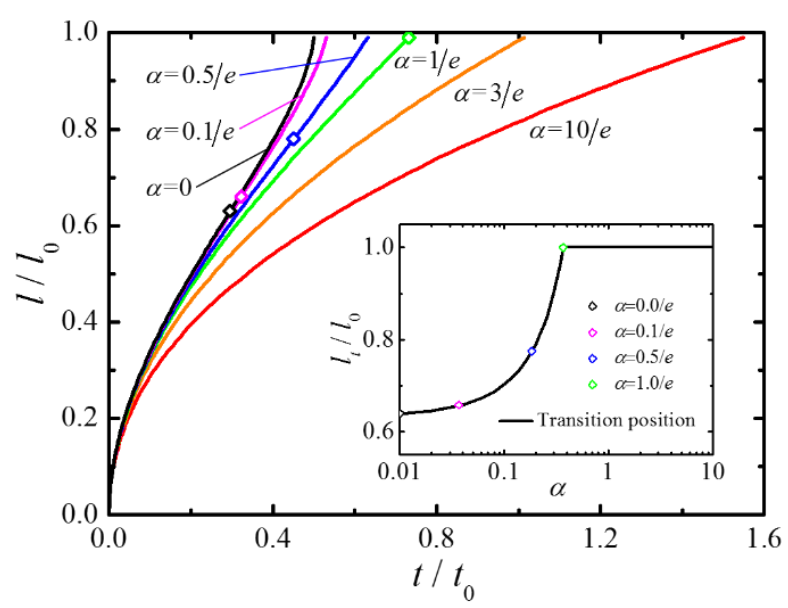

FIG. 3. Variations of the normalized distance against the normalized time for asymmetric capillary penetration with different geometrical shapes, the diamond symbols refer to the transition position of the penetration velocity. Insert: The normalized velocity transition position as a function of the geometrical factors.

Variation of the normalized capillary penetration distance with normalized time for six geometries $(\alpha=0,0.1 / e, 0.2 / e, 0.5 / e$, $1 / e, 3 / e$ and $10 / e$, with $e$ being Euler's number) are plotted in Fig. 3. As is evident from the inflection of the plotted curves, the penetration velocity transitions from a decreasing to an increasing trend during the penetration process for samples with $\alpha \leq 1 / e$. The transition points are marked with diamond symbols for each line (see Fig. 3 ), and occur at increasing distances for $\alpha$ values from 0 to $1 / e$. When $\alpha>1 / e$, the transition disappears and the penetration velocity decreases throughout the entire process. The variation of the transition position with the geometrical factor is shown in the insert in Fig. 3.

The penetration velocity $v=d l / d t$ can be determined from Eq. (6). Noting that $d v / d t=0$ at the transition position $l_{t}$, the relationship between evaporation rate, geometrical factor and transition position can be obtained from Eq. (6), as 


$$
\frac{\dot{m}_{e}}{\dot{m}_{e u}^{c}}=\frac{2(\alpha-1)^{2}\left\{1+\ln \left[1+\frac{l_{t}}{l_{0}}(\alpha-1)\right]\right\}}{\left[1+\left(1+\frac{l_{t}}{l_{0}}(\alpha-1)\right)^{2}\right] \ln \left[1+\frac{l_{t}}{l_{0}}(\alpha-1)\right]-2\left[1+\frac{l_{t}}{l_{0}}(\alpha-1)\right]^{2} \ln \left[1+\frac{l_{t}}{l_{0}}(\alpha-1)\right]^{2}-(\alpha-1) \frac{l_{t}}{l_{0}}\left[2+\frac{l_{t}}{l_{0}}(\alpha-1)\right]},
$$

where $\dot{m}_{e u}^{c}=P_{c} \cdot k \rho H / \mu l_{0}^{2}$ is a scaling parameter used to make the evaporation rate dimensionless. For negligible evaporation, the above equation reduces to an explicit expression for the transition position as

$$
\frac{l_{t}}{l_{0}}=\frac{1-\mathrm{e}^{-1}}{1-\alpha}
$$

This equation relates the transition point to the geometrical factor as shown in the insert of Fig. 3.
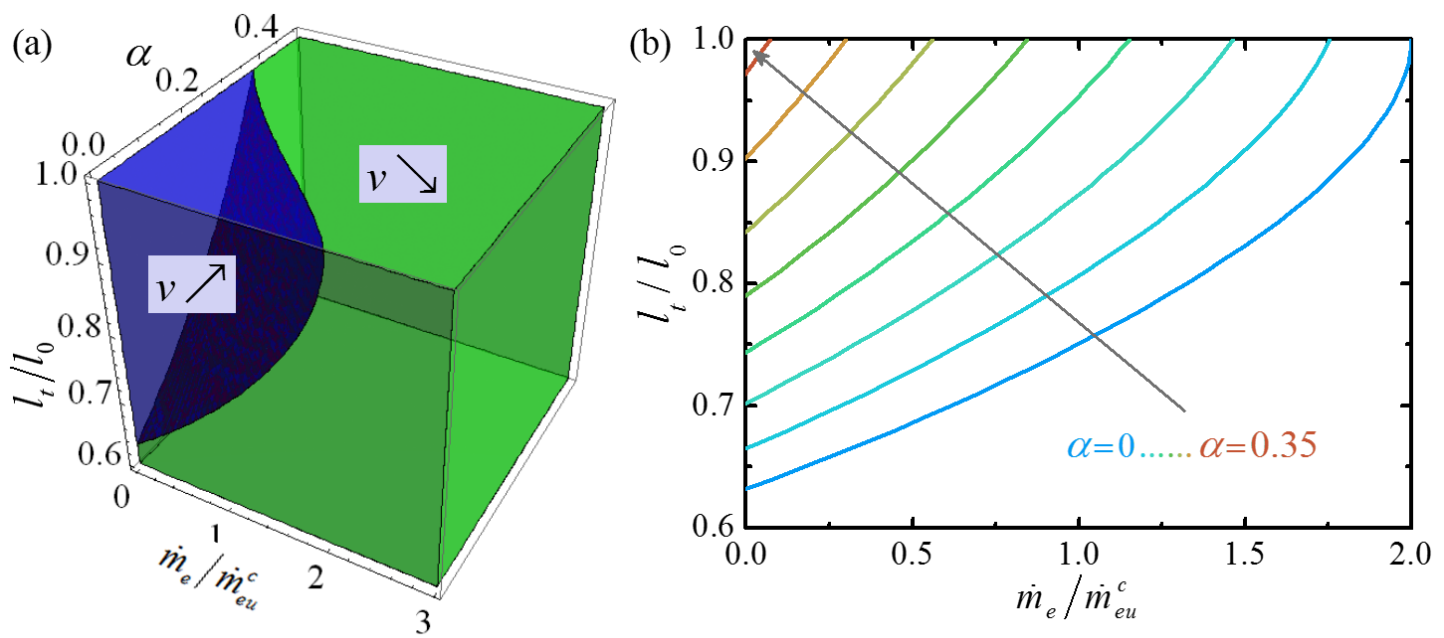

FIG. 4. (a) The distribution of the penetration velocity (acceleration or deceleration) under different evaporation conditions and geometrical shapes; (b) Transition position of the penetration velocity plotted versus evaporation rate for different geometrical shapes.

When the evaporation effect is nonnegligible, by using Eq. (9), the transition position $l_{t} / l_{0}$ can be predicted as a function of $\dot{m}_{e} / \dot{m}_{e u}^{c}$ and $\alpha$, as illustrated in Fig. 4a. In the blue region, the penetration exhibits acceleration, while the decelerating penetration is described by the green region. The interface between these two regions presents the transition position of penetration velocity $l_{t} / l_{0}$ and its dependence on parameters $\dot{m}_{e} / \dot{m}_{e u}^{c}$ and $\alpha$, which is predicted quantitatively by Eq. (9). In this 3D phase diagram, the interface between deceleration and acceleration reduces to a linear boundary when evaporation is negligible, which has been discussed before and given in a special case as shown in the insert in Fig. 3.

In order to more clearly show the transition position of penetration velocity for different 
geometry and evaporation conditions, $l_{t} / l_{0}$

is plotted as a function of $\dot{m}_{e} / \dot{m}_{e u}^{c}$ for different values of $\alpha$ in Fig. 4b. It can be found that the transition to accelerating penetration occurs at higher positions for higher evaporations rates for different geometries. Furthermore, for a given evaporation rate, the transition position is higher for larger geometrical factors. Based on such charts, the transition position can be readily determined for analytical purposes, and for informing the design of porous structures, such as chemical detection devices with controllable reaction times applied at different temperatures [5]. It should be mentioned that tuning the penetration velocity, especially the acceleration is challenging and necessitates the informed control of sample properties and environmental conditions.

\subsection{Evaporation limited capillary penetration}

As previously mentioned, the evaporation effect acts as a viscous resistance to the liquid front moving in the penetration process $[40,47]$. When the evaporation-induced viscous pressure loss equals the capillary pressure (viz., $P_{c}=P_{m}$ ), the velocity of liquid front reduces to zero, and the penetration will be restricted to a limited region with a critical length (see Fig. 2). By considering the critical condition of $P_{c}=P_{m}$, the critical length $l_{c}$ can be obtained as

$\left(\frac{l_{c}}{l_{0}}+\frac{1}{\alpha-1}\right)^{2} \ln \left[1+\frac{l_{c}}{l_{0}}(\alpha-1)\right]-\frac{l_{c}}{2 l_{0}}\left(\frac{2}{\alpha-1}+\frac{l_{c}}{l_{0}}\right)=\frac{\dot{m}_{e u}^{c}}{\dot{m}_{e}}$

where $\dot{m}_{e u}^{c}=P_{c} \cdot k \rho H / \mu l_{0}^{2}$ is the critical evaporation rate for a uniform porous plate that allows penetration through the entire material. This value has been used as a scaling parameter to normalize the evaporation rate in Eq. (9). Furthermore, if we focus on a special circumstance of the critical length exactly equaling to the length of the plate, i.e., $l_{c}=l_{0}$, the critical evaporation rate $\dot{m}_{e}^{c}$ can be given as

$\frac{\dot{m}_{e}^{c}}{\dot{m}_{e u}^{c}}=\frac{2(1-\alpha)^{2}}{1-\alpha^{2}+2 \alpha^{2} \ln \alpha}$.

This equation reveals the relation between $\dot{m}_{e}^{c}$ and $\dot{m}_{e u}^{c}$. The right hand side will reduce to 1 while $\alpha=1$, meaning $\dot{m}_{e}^{c}=\dot{m}_{e u}^{c}$ for the case of a uniform porous plate.

According to Eq. (11), the critical length of the evaporation-limited penetration region $l_{c} / l_{0}$ is plotted as a function of the evaporation rate $\dot{m}_{e} / \dot{m}_{e u}^{c}$ and geometrical factor $\alpha$ in Fig. 5a. It is clear that when evaporation rate is smaller than a critical value, i.e., $\dot{m}_{e} \leq \dot{m}_{e}^{c}$, the critical length remains the length of the sample, which means the liquid can penetrate the whole region. However, the critical length decreases from $l_{0}$ and approaches 0 when $\dot{m}_{e}>\dot{m}_{e}^{c}$. Note that there is a boundary at the top surface in Fig. 5a, as shown by a blue line, which delineates $l_{c}=l_{0}$ at critical conditions of evaporation rate and 
geometrical factor, and can be quantitatively described by Eq. (12) as shown in Fig. 5 b. Figure 5 indicates that the limited penetration region can be tuned by controlling the evaporation rate and geometry of the sample.
A tunable penetration region allows for optimized distribution of chemical reagents in a specific test zone in detection devices [34].
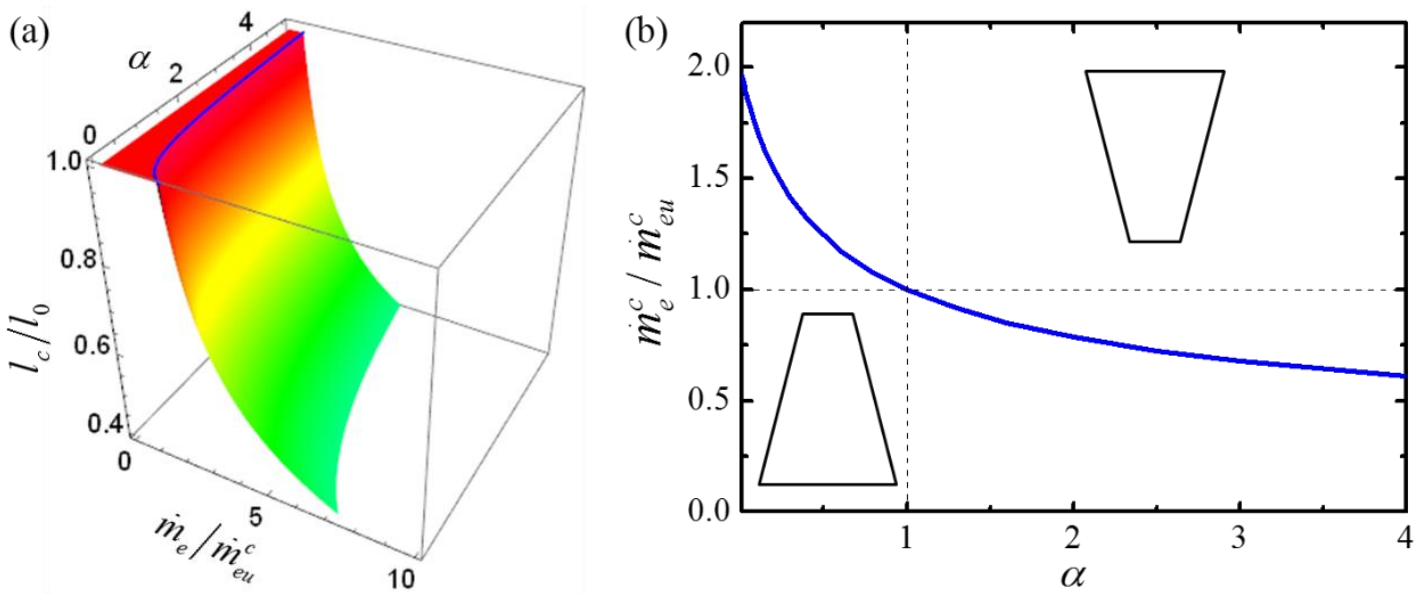

FIG. 5. (a) Normalized critical penetration distance versus normalized evaporation rate for asymmetric capillary penetration with different geometrical shapes; (b) Normalized critical evaporation rate as a function of geometrical factor.

It should be noted that all the results are given in a dimensionless form in the above analysis. Particularly, the evaporation rate $\dot{m}_{e}$ is normalized by a critical value $\dot{m}_{e}^{c}\left(\right.$ or $\dot{m}_{e u}^{c}$ ). According to the expression of $\dot{m}_{e}^{c}$ (i.e., Eq. (12)), we know that it depends on the properties of the liquid and the porous medium, and the geometrical parameters of the porous media. Moreover, the physical properties of a liquid (i.e., surface tension, density and viscosity) are influenced by environmental conditions, including temperature and vapor pressure [40, 44, 47]. In order to investigate the effects of these factors on the critical rate of evaporation, variations of the critical evaporation rate against the length of the sample and temperature are plotted in Fig. 6a and 6b, respectively. Two types of commonly used liquid, i.e., water and hydrofluoroether (HFE-7500), are considered. For each case, three different geometries, $\alpha=0.5,1$, and 1.5, are taken into account. The layer thickness is set to be $H=0.1 \mathrm{~mm}$. Other parameters of the porous medium, water and HEF-7500 under different temperatures are retrieved from Fries et al., ${ }^{40}$ Vargaftik et al. [49] and Rausch et al. [50], respectively. Figure 6 represents the linear relation between critical evaporation rate and the sample size and temperature. These findings demonstrate how liquid penetration can be tuned by changing ambient conditions, adopting different liquids and controlling porous media geometries. 

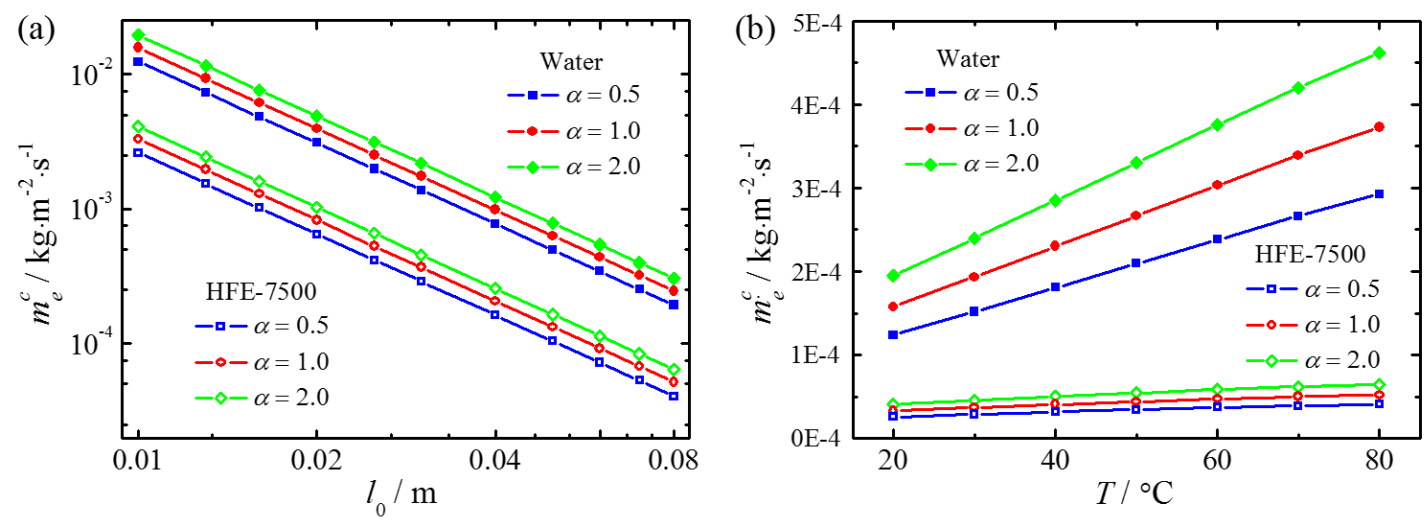

FIG. 6. Variation of the critical evaporation rate against (a) length of the sample at $T=20^{\circ} \mathrm{C}$ and (b) temperature with $l_{0}=0.1 \mathrm{~m}$ for porous plates with different geometries.

\subsection{Geometry-based compensation of penetration time}

In the above analyses, we have found that the penetration process is affected by not only liquid evaporation but also the geometry of the porous media. On one hand, the penetration process will be retarded by evaporation, on the other hand, changing the geometrical shape of the porous sample affects penetration in both accelerating and decelerating regimes. It indicates that the evaporation-retarded penetration can be compensated by choosing a proper geometrical shape of the porous sample. In other words, we can tune the capillary penetration in porous media by combining the geometry and evaporation effects. This inspires us to design suitable porous structures for particular using environment.
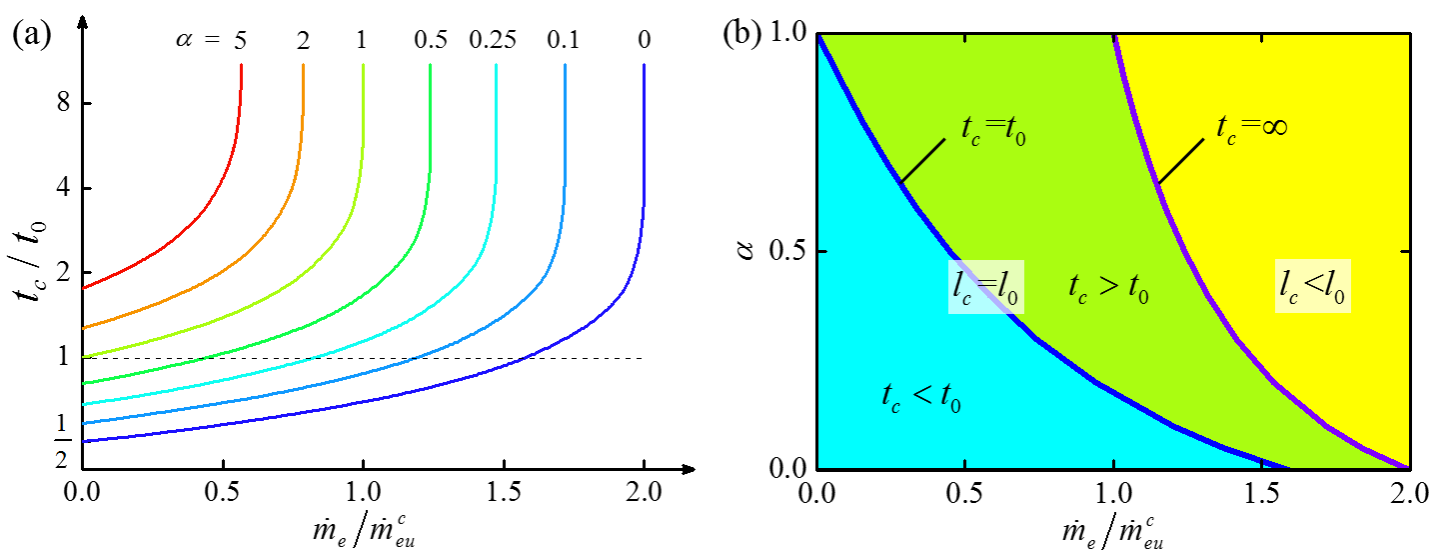

FIG. 7. (a) The critical penetration time versus evaporation rate for the penetration in porous plate with different geometrical shapes; (b) The coordination condition between geometrical factor and evaporation effect for compensating the evaporation-retarded penetration time.

For capillary penetration in porous media, the total penetration time is an important parameter attracting significant attention in industrial applications such as chemical analysis [37, 38]. As shown in Fig. 7a, the normalized critical time for penetration in the whole region (i.e., $t_{c} / t_{0}$, where $t_{0}$ is the critical penetration time of a uniform porous plate without evaporation) is plotted as function of normalized evaporation rate for samples with different geometries. It is clear 
that the penetration time increases with increasing evaporation rate for each geometrical factor, and approaches infinity when the evaporation rate tends to a critical value. After exceeding this critical evaporation rate, the penetration will be restricted to a limited region within the sample. The dotted line in Fig. 7a, which extends horizontally at $t_{c} / t_{0}=1$, reveals that tailoring the geometrical shape of a porous sample can compensate for the evaporation-retarded penetration.

To further understand this compensation mechanism, as shown in Fig. 7b, we plot the distribution of penetration time in a phase diagram in the space of $\dot{m}_{e} / \dot{m}_{e u}^{c}$ and $\alpha$. It can be seen that there are three regions in two main parts, i.e., the complete penetration part (i.e., $l_{c}=l_{0}$, the liquid can penetrate into the whole sample, including the shaded blue and cyan regions) and the incomplete penetration part (i.e., $l_{c}<l_{0}$, the liquid is limited to a finite region, see the shaded yellow region), with a purple solid line boundary, which indicates the limiting condition of complete penetration with infinite time (i.e., $t_{c}=\infty$ ). In the complete penetration region, there is a boundary between the blue and cyan regions shown as a green solid line, which is transplanted from the dotted line in Fig. 7a, and gives the coordination condition for the geometrical shape compensating the evaporation-retarded penetration time to be a constant (i.e., $t_{c}=t_{0}$ ). In the blue region, the total penetration time $t_{c}$ under the combination of evaporation rate and geometrical factor is smaller than the critical value $t_{0}$ (i.e., $\left.t_{c}<t_{0}\right)$. Thus, this region can be considered as an overcompensated region. In contrast, under-compensation is found in the cyan region, that is, the total penetration time is larger than the critical value, i.e., $t_{c}>t_{0}$.

In practical applications, the reference state for compensation may not always be the uniform porous plate. For any given reference state, the coordination condition of $\alpha$ and $\dot{m}_{e} / \dot{m}_{e u}^{c}$ can be obtained by moving the dotted line in Fig. 7a up (for the case of $\alpha>1$ ) or down (for $\alpha<1$ ). Accordingly, the position of the green solid line in the phase diagram in Fig. $7 \mathrm{~b}$ also needs to be adjusted corresponding to the dotted line. This compensation mechanism can be used as a basis to precisely control penetration processes in chemical analytical devices, with implications towards improving their accuracy $[34,48]$.

\section{Evaporation effect on the capillary flow in $3 D$ porous structures}

In the previous sections, by applying the proposed theoretical model, the combined effects of geometry and evaporation on the capillary penetration in 2D porous structures are investigated systematically. Nevertheless, capillary flow processes are also commonly found in 3D porous structures, such as capillary evaporators, drug delivery systems, and construction and geotechnical structures $[10,11,45]$, and most of the $3 \mathrm{D}$ porous structures with non-uniform cross sections [27]. Here we extend the proposed theoretical model to cover 3D porous structures and investigate the interplay of sample geometry and liquid evaporation 
during the capillary flow process.

\subsection{Theoretical model for capillary flow in 3D structures}

As shown in Fig. 8, we choose a hollow circular frustum cone (see Fig. 8a) and a hollow square frustum pyramid (see Fig. 8b) as two examples of 3D porous structures. When the porous samples attach with liquid reservoir, the liquid will transfer to the other end from the reservoir driven by the capillary pressure. For the hollow circular frustum, it is clear that the structure is axisymmetric. Its intermediate cross-section, i.e., the plane of symmetry, is an isosceles trapezoid. According to the symmetry, half of the intermediate cross-section, i.e., $\overline{O_{0} O_{1} Q_{1} Q_{0}}$, can be illustrated in Fig. 8c. For the hollow square frustum, the axisymmetry is not valid, but half of symmetrical plane $\overline{O_{0} O_{1} Q_{1} Q_{0}}$ can also be chose to characterize the basic geometric features of the whole structure. Similar to the previous analysis of 2D cases, half of the width and the porous layer thickness are assumed to vary with the position as

$$
R(z)=R_{0}\left[1-\frac{z}{h_{0}}\left(1-\alpha_{R}\right)\right],
$$

and

$T(z)=T_{0}\left[1-\frac{z}{h_{0}}\left(1-\alpha_{T}\right)\right]$,

where $R_{0}$ and $T_{0}$ are the half width and layer thickness at the edge in contact with the reservoir, respectively, $h_{0}$ is the length of the central axis of the hollow circular frustum cone and the hollow square frustum pyramid, $z$ is the local position in the penetrated region along the central axis, and $\alpha_{R}$ and $\alpha_{T}$ are the geometric parameters with respect to the half width and layer thickness, respectively. Similar to the 2D trapezoidal porous plate, the geometric feature of these 3D porous sample can be characterized by the geometric parameters $\alpha_{R}$ and $\alpha_{T}$.
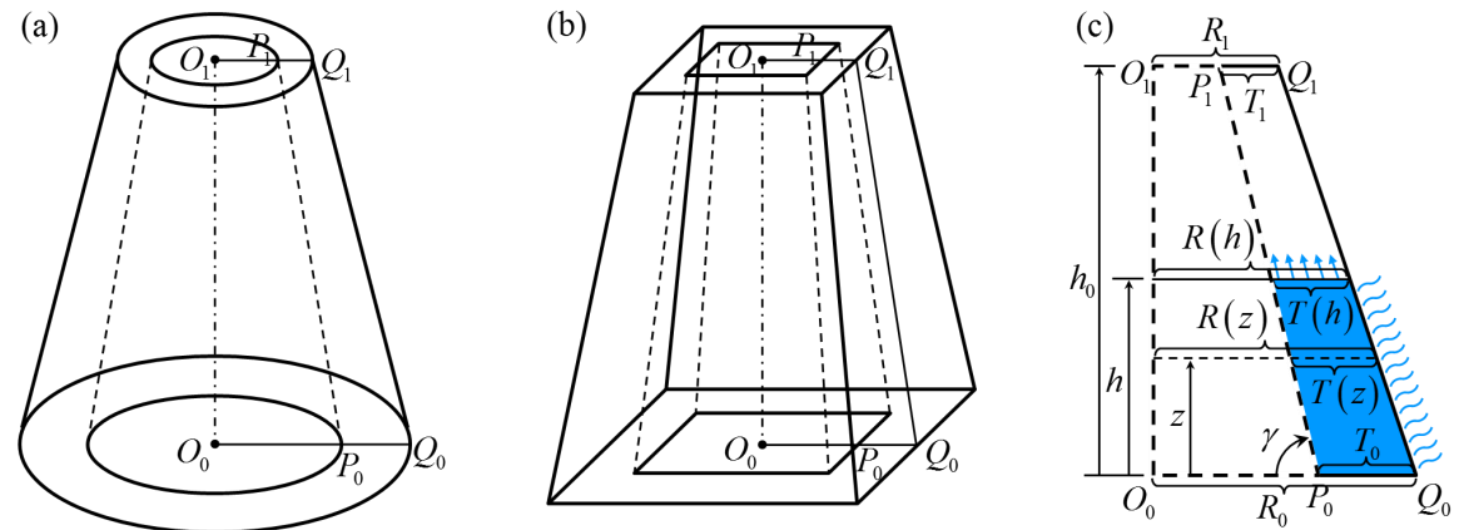

FIG. 8. Schematic illustration of 3D porous structures: (a) hollow circular frustum, (b) hollow square frustum, and (c) Intermediate cross-section of the 3D porous structures with capillary penetration incorporating evaporation effect.

For these 3D hollow porous structures, the liquid front is in general not a flat surface (i.e., 
a straight line in $2 \mathrm{D}$ sample) perpendicular to the flow direction but a curve surface, similar to the 2D trapezoidal structures. Specifically, for the hollow circular frustum (see Fig. 8(a)), the liquid front is axisymmetric about $\mathrm{z}$ axis, thus the 1D assumption of flat liquid front (see Fig. 8c) is valid when the opening angle is not large enough. For the hollow square frustum (see Fig. 8(b)), it should be noted that the 1D assumption will induce larger error, but it can be adopted for the theoretical model to investigate the basic characteristics

$$
\begin{aligned}
\dot{z} \cdot \pi\left[R^{2}(z)-(R(z)-T(z))^{2}\right] \phi & =\dot{h} \cdot \pi\left[R^{2}(h)-(R(h)-T(h))^{2}\right] \phi \\
& +\frac{\dot{m}_{e}}{\rho} \cdot \pi \sqrt{(h-z)^{2}+[R(z)-R(h)]^{2}} \cdot[R(z)+R(h)]
\end{aligned}
$$

where $z$ and $h$ indicate the local position in the penetrated region and the liquid front position, respectively, as shown in Fig. 8(c).

Technically, one can obtain the fully control equation for the moving liquid front position by combining Eqs. (5) and (15). However, it should be noted that the pressure gradient, which is used to determine the flow rate in Eq. (5), is depending on the flow direction. It is clearly seen from Fig. $8 \mathrm{c}$ that the flow direction is not along the central axis (i.e., $z$ axis) anymore, only if the hollow structure becomes solid. Here, it is assumed that the flow direction is along the inner boundary of the porous layer, i.e., $\overline{P_{0} P_{1}}$ in Fig. 8c. Introducing the slant angle $\gamma$, Eq. (5) can be rewritten, and the control equation can be obtained by combining with Eq. (15) as

$$
\begin{aligned}
P_{c}^{\prime}=\frac{\phi \mu}{k} \int_{0}^{h} \dot{z} d z & =\frac{\phi \mu}{k} \int_{0}^{h} \frac{2 R(h) T(h)-T(h)^{2}}{2 R(z) T(z)-T(z)^{2}} \cdot \dot{h} d z \\
& +\frac{\phi \mu}{k} \cdot \frac{\dot{m}_{e}}{\rho \phi} \int_{0}^{h} \frac{[R(z)+R(h)] \cdot \sqrt{(h-z)^{2}+[R(z)-R(h)]^{2}}}{2 R(z) T(z)-T(z)^{2}} d z
\end{aligned}
$$

where $P_{c}^{\prime}=P_{c} \cdot(\sin \gamma)^{2}$, in which $P_{c}$ is the capillary force with the same expression as $2 \mathrm{D}$ case as $P_{c}=2 \sigma \cos \theta_{s} / R_{\text {eff }}$.

By substituting the expressions of $R(z), T(z), R(h)$ and $T(h)$, through Eqs. (13) and (14), into Eq. (16), the penetration distance, i.e., the liquid front position, can be calculated as a function of penetration time. It should be noted that the mass conservation of the hollow square frustum (see Fig. 8(b)) has the similar expression as Eq. (15) for the hollow circular frustum, and we can further confirm that Eq. (16) is also valid for the hollow square frustum. 
Noted that there are two integral functions in Eq. (16), the explicit expression is not easy to obtain for general case. Here we focus on two special cases, i.e., uniform thickness and varying thickness, to derive the explicit expressions of control equation. When the 3D hollow porous structure has a uniform thickness, i.e., $T(z)=T_{0}$, the geometrical parameters can be set to $\alpha_{R}=\alpha$ and $\alpha_{T}=1$. Following these conditions, the control equation can be obtained as

$$
\begin{aligned}
P_{c} & =\frac{\phi \mu l_{0}^{2}}{k} \cdot \frac{1-\Pi / 2-(1-\alpha) \bar{h}}{1-\alpha} \ln \left[\frac{1-\Pi / 2}{1-\Pi / 2-(1-\alpha) \bar{h}}\right] \cdot \frac{d \bar{h}}{d t} \\
& +\frac{\mu \dot{m}_{e} l_{0}^{2}}{2 \rho k T_{0}^{\prime}} \cdot\left\{\frac{[1-(1-\alpha) \bar{h}]^{2}-\Pi^{2} / 4}{(1-\alpha)^{2}} \ln \left[\frac{1-\Pi / 2-(1-\alpha) \bar{h}}{1-\Pi / 2}\right]-\frac{\bar{h}^{2}}{2}+\frac{(1+\Pi / 2) \bar{h}}{1-\alpha}\right\}
\end{aligned}
$$

where $l_{0}$ is the distance of the sample along the flow direction and can be related to the axial length $h_{0}$ via $h_{0} / l_{0}=\sin \gamma . \bar{h}=h / h_{0}$ is the projected length of the penetrated region, $T_{0}^{\prime}=T_{0} / \sqrt{1+(1-\alpha)^{2} / \mathrm{H}^{2}}$ is the effective thickness of the porous layer at the bottom surface, and $\Pi=T_{0} / R_{0}$ is the relative thickness with respect to the half width. Following the flat front assumption, one can find that $\bar{l}=l / l_{0}=\bar{h}$, where $l$ is the length of the penetrated region along the flow direction.

When the thickness of the porous layer is much smaller than the width of the sample, i.e., $\Pi \rightarrow 0$, hollow square frustum pyramid can be unfolded as four trapezoids. Correspondingly, Eq. (17) will reduce to Eq. (6) by replacing $\bar{h}$ as $\bar{l}$. Furthermore, hollow circular frustum cone can be unfolded as a sector ring when $\Pi \rightarrow 0$. Hence Eq. (17) can reduce to the theoretical model for radial penetration in our previous work [47], by replacing $\bar{h}$ as $\bar{r}$ through the relation $\bar{r}=1-\bar{h} \cdot(1-\alpha)$.

For the special case of equal proportional thickness, i.e., $\alpha_{R}=\alpha_{T}=\alpha$, the control equation can be derived as

$$
P_{c}=\frac{\phi \mu l_{0}^{2}}{k} \cdot[1-(1-\alpha) \bar{h}] \bar{h} \cdot \frac{d \bar{h}}{d t}+\frac{\mu \dot{m}_{e} l_{0}^{2}}{2 \rho k T_{0}^{\prime}} \cdot \frac{\bar{h}^{2}}{1-\Pi / 2} .
$$

In this case, when $\Pi=1$, the hollow structure becomes solid. Furthermore, when $\alpha=1$, Eq. (18) will reduce to the classical 1D model given by Fries et al. [40]. 
According to the developed theoretical models, i.e., Eqs. (17) and (18), the capillary penetration process in $3 \mathrm{D}$ porous structures can be predicted for samples with different geometrical shapes and under different evaporation conditions. Here we focus on the hollow porous sample with uniform layer thickness as an example. As shown in Fig. 9, the normalized penetration distances are plotted as a function of normalized penetration time for two sets of geometric parameters, i.e., $\alpha=2.0$ and $\alpha=0.5$. For each geometry, three samples with different relative layer thickness, i.e., $\Pi=0.01 \bar{\alpha}, 0.5 \bar{\alpha}$ and $1.0 \bar{\alpha}$ (where $\bar{\alpha}=\alpha \quad$ when $\quad \alpha<1 \quad$ and $\quad \bar{\alpha}=1 \quad$ when $\alpha \geq 1$ ), are considered, as shown by solid lines with different colors. For the purpose of comparison, the prediction of 2D model (Eq. (6)) are also plotted by symbols and definitely consist with the 3D case of $\Pi=$ $0.01 \bar{\alpha}$.
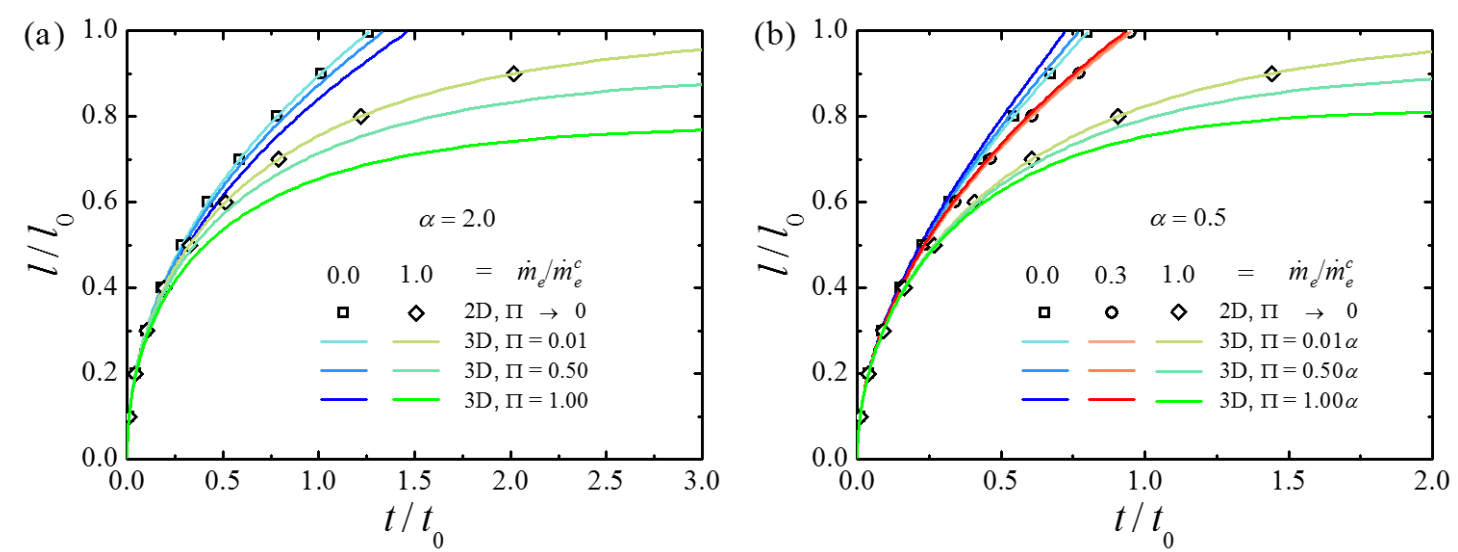

FIG. 9. Normalized penetration distance versus penetration time for capillary penetration in hollow porous sample (lines) with: (a) $\alpha=2.0$ (under evaporation rates $\dot{m}_{e} / \dot{m}_{e}^{c}=0.0$ and 1.0) and (b) $\alpha=0.5\left(\dot{m}_{e} / \dot{m}_{e}^{c}=0.0,0.3\right.$ and 1.0) with relative layer thickness $\Pi=0.01 \bar{\alpha}, 0.5 \bar{\alpha}$ and $1.0 \bar{\alpha}$. The results of 2D model prediction (symbols) are also included for the purpose of comparison.

It can be seen from Fig. 9 that, for both two sets of geometries, the evaporation of liquid weaken the penetration process generally, i.e., higher evaporation rate leads to slower flow velocity for all the three samples with different relative thickness. However, for a given evaporation condition, the effect of relative layer thickness on the penetration will depend on the geometric shape of the sample. Specifically, for the case of $\alpha=2.0$ under two evaporation rates (i.e., $\dot{m}_{e} / \dot{m}_{e}^{c}=0$ and 1.0), as shown in Fig. 9(a), slower flow velocity can be found for the sample with larger relative thickness. However, for the case of $\alpha=0.5$, it is interesting to find that there is a transition of the effect of relative layer thickness on the penetration from negative to positive by varying the evaporation rates, see Fig. 9(b).

When $\dot{m}_{e} / \dot{m}_{e}^{c}=0$, slower flow velocity is found for the thicker sample, but on the contrary, faster flow velocity is obtained in the thinner sample when $\dot{m}_{e} / \dot{m}_{e}^{c}=1$. The transition is approximately occurred at $\dot{m}_{e} / \dot{m}_{e}^{c}=0.3$, and the flow velocity is not 
dependent on the relative layer thickness at this special evaporation condition. This interesting transition phenomenon will have significant effect on the compensation mechanism in 3D conditions.

\subsection{Compensation mechanism in 3D conditions}

As we have discussed in Section 4.3, the evaporation-retarded penetration can be compensated by tailoring geometrical shape of the porous sample. For the $2 \mathrm{D}$ porous sample, only the geometric factor is tunable, but for the 3D porous sample, one more parameter (i.e., the relative layer thickness) will be introduced to increase the design flexibility. Here we also choose the critical penetration time for the whole region $\left(t_{c}\right)$ as an object variable of the compensation, and the corresponding reference is the critical penetration time of a uniform porous cylinder without evaporation $\left(t_{0}\right)$.
First, we focus on the 3D hollow porous structure with uniform thickness. As shown in Fig. 10, the normalized critical penetration time (i.e., $t_{c} / t_{0}$ ) is plotted as a function of evaporation rate for porous samples with different geometrical shapes (i.e., $\alpha=0,0.1$, $0.25,0.5,1.0$ and 2.0). For each geometry, three samples with different relative thickness, i.e., $\Pi=0.01 \bar{\alpha}, 0.5 \bar{\alpha}$ and 1.0 $\bar{\alpha}$, are considered. When the relative layer thickness approaches zero (e.g., $\Pi=0.01$ $\bar{\alpha})$, the results of $3 \mathrm{D}$ cases are consistent with the $2 \mathrm{D}$ results (as shown by symbols). Similar as the 2D cases (see Fig. 7(a)), the penetration time increases with increasing evaporation rate for each sample with different geometric factor and relative thickness. Additionally, the penetration time approaches infinity when the evaporation rate tends to a critical value, and the limited penetration will occur when the evaporation rate exceeds the critical value.

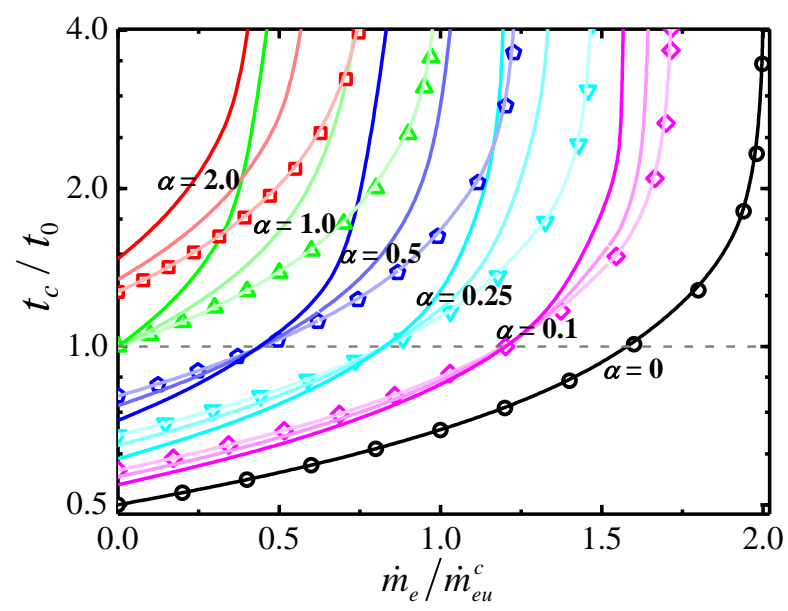

FIG. 10. The critical penetration time versus evaporation rate for the penetration in $3 \mathrm{D}$ porous samples (lines) with different geometrical shapes (i.e., $\alpha=0,0.1,0.25,0.5,1.0$ and 2.0). The results of 2D model prediction (symbols) are also incorporated as comparison.

For the samples with given geometric factor, the relation between critical penetration time and evaporation rate is dependent upon the relative layer thickness of the sample. However, it can be interestingly found that this dependence vanishes at the state of 
$t_{c} / t_{0}=1$, which reveals that the

coordination condition of compensation for the evaporation-retarded penetration is not depending on the relative layer thickness. By extracting the intersection point at the horizontal dotted line in Fig. 10, the compensation condition for 3D porous structures can be plotted in Fig. 11(a). This condition is corresponding to the phase boundary line in left side of Fig. 7(b) for the 2D compensation. Similar as the 2D case, for a given 3D porous structure, when the

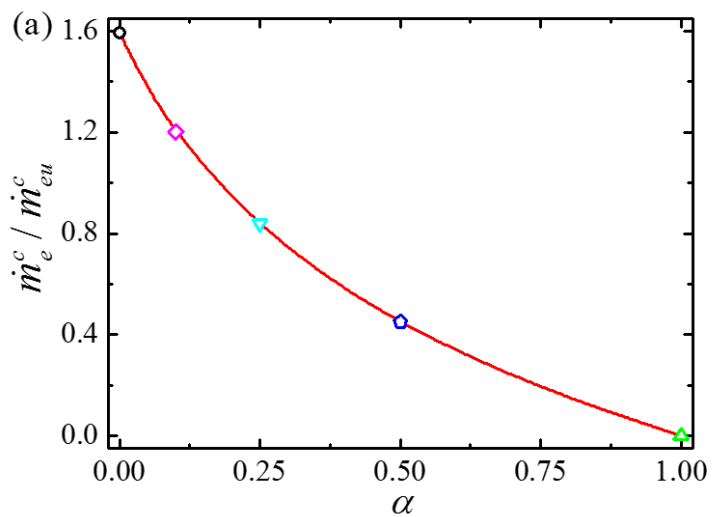

condition of evaporation and geometric shape situate in the overcompensated region, which is located in the left side of the red line, the total penetration time $t_{c}$ is smaller than the critical value $t_{0}$ (i.e., $\left.t_{c}<t_{0}\right)$. On the contrary, the larger total penetration time (i.e., $t_{c}>t_{0}$ ) can be found in the undercompensation region, which is located in the right side of the red line.

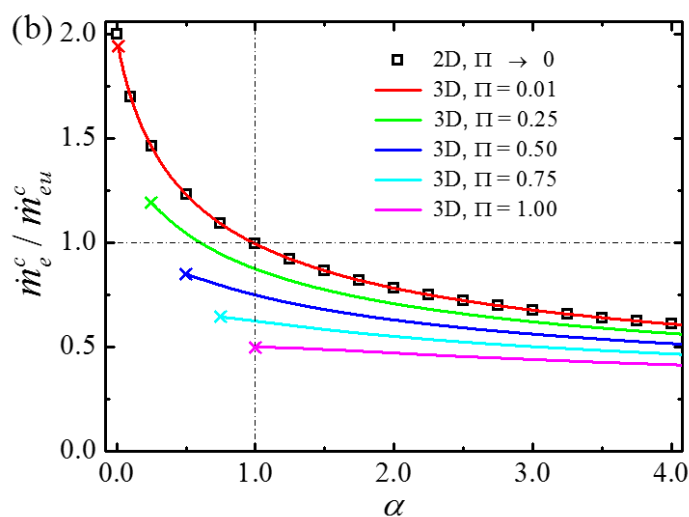

FIG. 11. (a) The coordination condition between geometrical factor and evaporation effect for compensating of the evaporation-retarded penetration time; (b) Normalized critical evaporation rate for the complete penetration in 3D porous sample with different relative layer thickness (i.e., $\Pi=0.01,0.25,0.50,0.75$ and 1.00 ) are plotted as a function of geometrical factor (lines). The critical condition for the complete penetration in 3D sample (symbols) are also incorporated as compression.

When the evaporation rate is larger enough, the liquid penetration will be limited in a finite region. By employing the equilibrium condition of $P_{c}=P_{m}$, the critical condition for the complete penetration (i.e., the liquid can penetrate into the whole sample exactly) can be obtained as

$\frac{\dot{m}_{e u}^{c}}{\dot{m}_{e}^{c}}=\frac{\alpha^{2}-\Pi^{2} / 4}{(1-\alpha)^{2}} \ln \left[\frac{\alpha-\Pi / 2}{1-\Pi / 2}\right]-\frac{1}{2}+\frac{1+\Pi / 2}{1-\alpha}$.

It can be found that, when the relative layer thickness $\Pi \rightarrow 0$, Eq. (19) will reduce to Eq. (12), which is governing the critical condition for the complete penetration in 2D porous samples. By applying Eq. (19), the normalized critical evaporation rate for the complete penetration in 3D porous sample can be plot as a function of geometrical factor in Fig. 11(b). Five cases with different relative thickness, i.e., $\Pi=0.01,0.25$, $0.50,0.75$ and 1.00, are considered here, and the case with $\Pi=0.01$ is consisting with the $2 \mathrm{D}$ results (as shown by symbols). Due to the relative thickness is confined with $\Pi \leq \bar{\alpha}$, it is clearly seen that the critical 
condition between geometrical factor and evaporation rate is truncated at $\alpha=\Pi$ for each case. Additionally, for a given geometrical factor, larger relative thickness corresponds to smaller critical evaporation rate, that means the complete penetration is easier to attain for the sample with larger relative thickness.

All the discussion presented above are focusing on the 3D hollow porous structures with uniform layer thickness. However, we

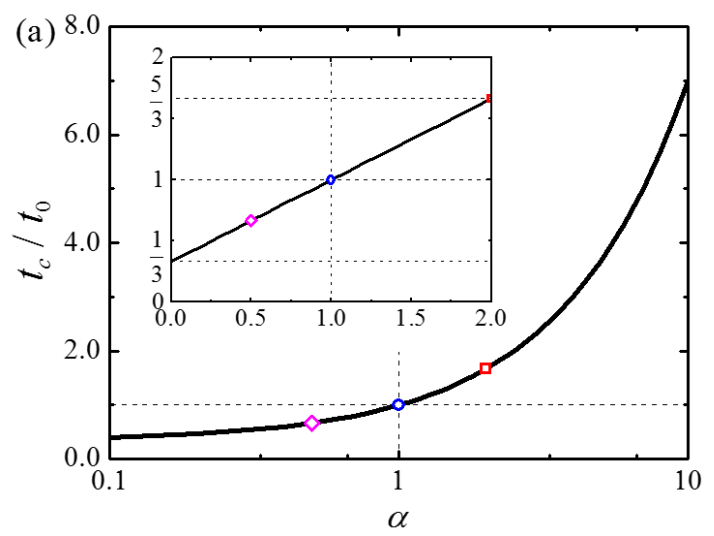

cannot access the properties of solid cylindrical porous sample by changing the geometrical parameters, even if the inner radial reduces to zero. To this end, here we consider the liquid penetration process in 3D hollow porous structures with proportional layer thickness. Applying the proposed model, i.e., Eq. (18), the variation of the liquid front with penetration time can be calculated for 3D hollow porous structure with different geometrical shape under different evaporation condition.

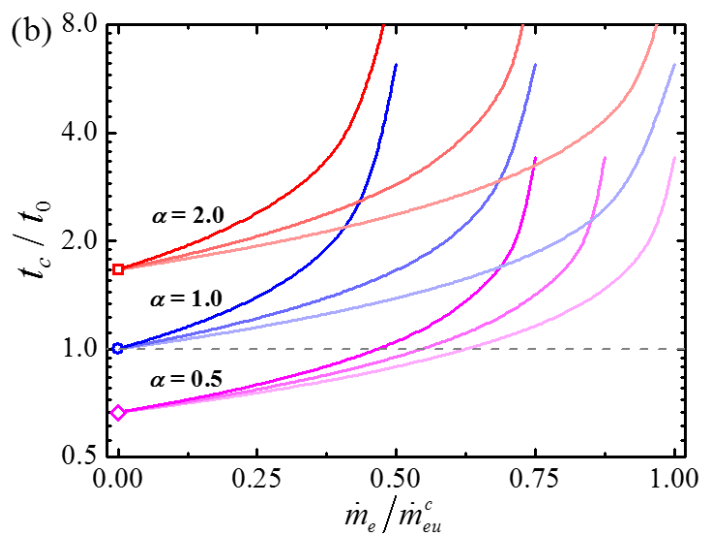

FIG. 12. The critical penetration time for the penetration in 3D porous samples versus (a) geometrical factor, and (b) evaporation rate for different geometrical shapes.

When the evaporation is negligible, i.e., $\dot{m}_{e}=0$, by integrating Eq. (18), the relation between penetration distance and penetration time can be obtained explicitly as $\bar{h}^{2}-2 / 3 \cdot(1-\alpha) \bar{h}^{3}=\bar{t} \quad$ with $\quad \bar{t}=t / t_{0}$.

For the complete penetration, i.e., $\bar{h}=1$, the critical condition can be further simplified as $1 / 3+2 \alpha / 3=\bar{t}_{c}$, where $\bar{t}_{c}=t_{c} / t_{0}$ is the normalized critical penetration time. The critical penetration time $\bar{t}_{c}$ is plotted as a function of the geometrical factor $\alpha$ in Fig. 12(a) with semi-log coordinates. The asymmetric capillary flow is clearly found when $\alpha$ varying from 0.1 to 10 . Moreover, the linear variation of $\bar{t}_{c}$ to $\alpha$ is also shown in the insert of Fig. 12(a) with initial value of $\overline{t_{c}}=1 / 3$ when $\alpha=0$.

When the evaporation is non-negligible, as we have discussed before, the evaporation restarted penetration can be compensated by changing the geometrical shape of the porous sample. For the 3D hollow porous structures with proportional layer thickness, the normalized critical penetration time can be plotted as a function of evaporation rate for samples with different geometrical shapes (i.e., $\alpha=0.5,1.0$ and 2.0), as shown in Fig. 12(b). Three samples with different relative 
thickness, i.e., $\Pi=0.01 \bar{\alpha}, 0.5 \bar{\alpha}$ and 1.0 $\bar{\alpha}$, are considered for each geometry. It is clearly seen that the relative thickness has no effect on the critical time when the evaporation is negligible (i.e., $\dot{m}_{e}=0$ ).

With the evaporation rate increasing, the significance of the effect of relative thickness on the critical time is increasing. Following the horizontal dotted line in Fig. 12(b), one can find that the coordination condition of compensation is depending on the relative layer thickness, which is different from the results of 3D structures with uniform layer thickness. Additionally, the critical condition of complete penetration is also depending on the relative thickness.

The findings presented in this section provide us more clear understanding about the capillary flow in 3D porous structures, especially the compensation mechanism in 3D conditions. By means of these findings, the design of capillary flow devices can be improved to facilitate the applications in a wide range, especially for the devices with high precision and sensitivity requirements.

\section{Conclusion}

In summary, a general framework has been developed to quantitatively investigate the significant effects of sample geometry and the liquid evaporation on capillary penetration processes in both $2 \mathrm{D}$ and $3 \mathrm{D}$ porous media with non-uniform cross sections. By combining the effects of geometry and evaporation, the velocity and extent of capillary penetration can be tuned with implications for diverse practical applications. Numerical simulations have been performed to support the developed theoretical model for 2D porous media. Good agreement is obtained for the base angles between $45^{\circ}$ and $135^{\circ}$.
For 2D porous media, the velocity of capillary penetration is found to be sensitive to the geometry of the porous sample, and it is found to transition from a decreasing to an increasing trend during the penetration process for a given geometry. Moreover, the distribution of penetration velocity can be modified by evaporation effects. The transition position has been predicted quantitatively as a function of the evaporation rate and the geometrical factor. Furthermore, a critical value of the evaporation was found to exist, above which liquid penetration is restricted to a limited region with a predictable boundary. The critical length of the limited penetration region depends on the evaporation rate and geometrical factor. It is particularly interesting to note that evaporation-retarded penetration can be compensated by choosing an appropriate sample geometry, and the coordination condition is given theoretically.

The capillary penetration in $3 \mathrm{D}$ porous structures was investigated. It is found that the relative layer thickness (uniform of variable) has a significant effect on the penetration process and the critical condition of the complete penetration in 3D structures. Another interesting finding is that the compensation condition for the evaporationretarded penetration in $3 \mathrm{D}$ porous structures with uniform thickness does not depend on the layer thickness, but such dependence is found for porous structures with proportional thickness.

The present analysis provides a useful framework to investigate the underlying mechanisms of penetration processes by combining geometry and evaporation effects. Insights gained from this work warrant new designs of more complex and actual porous 
architectures to achieve active control of the capillary penetration processes for a wide range of practical applications. Furthermore, it is worth mentioning that the present framework to consider geometrical factors can be applied to alternative shapes, other than the trapezoid and hollow circular/square frustum we discussed here.

\section{Acknowledgments}

The authors are grateful for the financial support of this work by the National Natural Science Foundation of China (No. 11472149 and 11672149) and the Tsinghua University Initiative Scientific Research Program (No. 2014z22074). M.L. acknowledges support from the Endeavour Research Fellowship founded by Australian Government. Y.G. acknowledges support from The University of Sydney SOAR Fellowship.

\section{Competing financial interests}

The authors declare no competing financial interests.

\section{References}

1. A. M. Miranda, I. L. Menezessobrinho, M. S. Couto, Spontaneous imbibition experiment in newspaper sheets. Phys. Rev. Lett. 104 (2010) 086101.

2. C. Hall, W. D. Hoff, Rising damp: capillary rise dynamics in walls. Proc. R. Soc. A 463 (2007) 1871-1884.

3. X. Li, D. R. Ballerini, W. Shen, A perspective on paper-based microfluidics: current status and future trends. Biomicrofluidics 6 (2012) 011301.

4. D. M. Cate, J. A. Adkins, J. Mettakoonpitak, C. S. Henry, Recent developments in paper-based microfluidic devices. Anal. Chem. 87 (2015) 19-41.

5. R. Tang, H. Yang, Y. Gong, M. You, Z. Liu, J. R. Choi, T. Wen, Z. Qu, Q. Mei, F. $\mathrm{Xu}, \mathrm{A}$ fully disposable and integrated paper-based device for nucleic acid extraction, amplification and detection. Lab Chip 17 (2017) 1270-1279.

6. N. Hashemi, J. M. Lackore, F. Sharifi, P. J. Goodrich, M. L. Winchell, N. Hashemi, A paper-based microbial fuel cell operating under continuous flow condition. Technology, 4 (2016) 98-103.

7. G. Xue, Y. Xu, T. Ding, J. Li, J. Yin, W. Fei, et al. Water-evaporation-induced electricity with nanostructured carbon materials. Nature Nanotech. 12 (2017) 317-321.

8. E. Kim, Y. N. Xia, G. M. Whitesides, Polymer microstructures formed by molding in capillaries. Nature 376 (1995) 581-584.

9. Y. Y. Liu, J. H. Xin, C. H. Choi, Cotton fabrics with single-faced superhydrophobicity. Langmuir 28 (2012) 17426-17434.

10. L. Mottet, T. Coquard, M. Prat, Three dimensional liquid and vapour distribution in the wick of capillary evaporators. Int. J. Heat Mass Transf. 83 (2015) 636-651.

11. M. I. M. Torres, V. P. D. Freitas, Treatment of rising damp in historical buildings: wall base ventilation. Build. Environ. 42 (2007) 424-435.

12. N. R. Morrow, G. Mason, Recovery of oil by spontaneous imbibition. Curr. Opin. Colloid Interface Sci. 6 (2001) 321-337.

13. J. Hyväluoma, P. Raiskinmäki, A. 
Jäsberg, A. Koponen, M. Kataja, J. Timonen, Simulation of liquid penetration in paper. Phys. Rev. E 73 (2006) 036705.

14. N. K. Palakurthi, S. Konangi, U. Ghia, K. Comer, Micro-scale simulation of unidirectional capillary transport of wetting liquid through $3 \mathrm{D}$ fibrous porous media: Estimation of effective pore radii. Int. J Multiphase Flow 77 (2015) 48-57.

15. A. Marmur, Kinetics of penetration into uniform porous media: testing the equivalent-capillary concept. Langmuir 19 (2003) 5956-5959.

16. E. Elizalde, R. Urteaga, R. R. Koropecki, C. L. Berli, Inverse problem of capillary filling. Phys. Rev. Lett. 112 (2014) 134502.

17. V. I. Nikitsin, B. Backiel-Brzozowska, Determining hydraulic radii of construction wall materials in capillary moisture transfer. Int. J. Heat Mass Transf. 88 (2015) 558-564.

18. S. F. Ni, K. Jessen, Theoretical analysis of capillary rise in porous Media. Transp. Porous Media 110 (2015) 141-155.

19. P. G. Gennes, F. Brochard-Wyart, D. Quere, Capillarity and Wetting Phenomena: Drops, Bubbles, Pearls, Waves, Springer, New York, 2004.

20. R. Lucas, Rate of capillary ascension of liquids. Kolloid-Z. 23 (1918) 15-22.

21. E. W. Washburn, The dynamics of capillary flow. Phys. Rev. 17 (1921) 273-283.

22. M. Lago and M. Araujo, Capillary rise in porous media. J. Colloid Interface Sci. 234, 35-43 (2001).

23. B. J. Mullins, R. D. Braddock, Capillary rise in porous, fibrous media during liquid immersion. Int. J. Heat Mass Transf. 55 (2012) 6222-6230.

24. D. Danino, A. Marmur, Radial capillary penetration into paper: limited and unlimited liquid reservoirs. J. Colloid Interface Sci. 166 (1994) 245-250.

25. M. Conrath, N. Fries, M. Zhang, M. E. Dreyer, Radial capillary transport from an infinite reservoir. Transp. Porous Media 84 (2010) 109-132.

26. J. Xiao, H. A. Stone, D. Attinger, Sourcelike solution for radial imbibition into a homogeneous semi-infinite porous medium. Langmuir $28 \quad$ (2012) 4208-4212.

27. M. Reyssat, L. Courbin, E. Reyssat, H. A Stone, Imbibition in geometries with axial variations. J. Fluid Mech. 615 (2008) 335-344.

28. K. Bal, J. Fan, M. K. Sarkar, L. Ye, Differential spontaneous capillary flow through heterogeneous porous media. Int. J. Heat Mass Transf. 54 (2011) 30963099.

29. Z. Sadjadi, H. Rieger, Scaling theory for spontaneous imbibition in random networks of elongated pores. Phys. Rev. Lett. 110 (2013) 144502.

30. X. Clotet, J. Ortín, S. Santucci, Disorderinduced capillary bursts control intermittency in slow imbibition. Phys. Rev. Let 113 (2014) 074501.

31. Y. Jin, X. Li, M. Zhao, X. Liu, H. Li, A mathematical model of fluid flow in tight porous media based on fractal assumptions. Int. J. Heat Mass Transf. 108 (2017) 1078-1088.

32. K. Zheng, T. Wang, H. Luo, Z. Gong, W. $\mathrm{Wu}$, Fractal analysis of flow resistance in 
random porous media based on the staggered pore-throat model. Int. J. Heat Mass Transf. 115 (2017) 225-231.

33. E. Fu, C. Downs, Progress in the development and integration of fluid flow control tools in paper microfluidics. Lab Chip. 17 (2017) 614-628.

34. J. R. Choi, K. W. Yong, R. Tang, Y. Gong, T. Wen, H. Yang, A. Li, Y. C. Chia, B. Pingguan-Murphy, F. Xu, Lateral flow assay based on paper-hydrogel hybrid material for sensitive point-of-care detection of dengue virus. Adv. Healthcare Mater. 6 (2017) 2192-2659.

35. S. Mendez, E. M. Fenton, G. R. Gallegos, D. N. Petsev, S. S. Sibbett, H. A. Stone, Y. Zhang, G. P.Lopez, Imbibition in porous membranes of complex shape: quasi-stationary flow in thin rectangular segments. Langmuir $26 \quad$ (2010) 1380-1385.

36. E. M. Benner, D. N. Petsev, Potential flow in the presence of a sudden expansion: application to capillary driven transport in porous media. Phys. Rev. E 87 (2013) 033008.

37. D. Shou, L. Ye, J. Fan, K. Fu, M. Mei, H. Wang, Q. Chen, Geometry-induced asymmetric capillary flow. Langmuir 30 (2014) 5448-5454.

38. D. Shou, L. Ye, J. Fan, K. Fu, Optimal design of porous structures for the fastest liquid absorption. Langmuir 30 (2014) 149-155.

39. E. Elizalde, R. Urteaga, C. L. Berli, Rational design of capillary-driven flows for paper-based microfluidics. Lab Chip 15 (2015) 2173-2180.

40. N. Fries, K. Odic, M. Conrath, M. Dreyer, The effect of evaporation on the wicking of liquids into a metallic weave. J. Colloid Interface Sci. 321 (2008) 118-129.

41. R. Wu, G. M. Cui, R. Chen, Pore network study of slow evaporation in hydrophobic porous media. Int. J. Heat Mass Transf. 68 (2014) 310-323.

42. Z. Liu, J. Hu, Y. Zhao, Z. Qu, F. Xu, Experimental and numerical studies on liquid wicking into filter papers for paper-based diagnostics. Appl. Therm. Eng. 88 (2015) 280-287.

43. M. Meshkat, R. C. Warner, S. R.Workman, Evaporation reduction potential in an undisturbed soil irrigated with surface drip and sand tube irrigation. Trans. ASAE 43 (2000) 79-86.

44. E. F. Médici, J. S. Allen, Evaporation, two phase flow, and thermal transport in porous media with application to lowtemperature fuel cells. Int. J. Heat Mass Transf. 65 (2013) 779-788.

45. L. Mottet, M. Prat, Numerical simulation of heat and mass transfer in bidispersed capillary structures: Application to the evaporator of a loop heat pipe. Appl. Therm. Eng. 102, (2016) 770-784.

46. M. Mercuri, K. Pierpauli, M. G. Bellino, C. L. A. Berli, Complex filling dynamics in mesoporous thin dilms. Langmuir 33 (2017) 152-157.

47. M. C. Liu, J. Wu, Y. X. Gan, D. A. H. Hanaor, C. Q. Chen, Evaporation limited radial capillary penetration in porous media. Langmuir 32 (2016) 9899-9904.

48. S. Jahanshahi-Anbuhi, P. Chavan, C. Sicard, V. Leung, S. Z. Hossain, R. Pelton, J. D. Brennan, C. D. Filipe, Creating fast flow channels in paper fluidic devices to control timing of 
Cite as: Liu, M., Wu, J., Gan, Y., Hanaor, D. A., \& Chen, C. Q. (2018). Tuning capillary penetration in porous media: combining geometrical and evaporation effects. International Journal of Heat and Mass Transfer, 123, 239-250.

sequential reactions. Lab Chip 12 (2012)

5079-5085.

49. N. B. Vargaftik, Y. K. Vinogradov, and V.

S. Yargin, Handbook of Physical Properties of Liquids and Gases, Begell House, New York, 1996.

50. M. H. Rausch, L. Kretschmer, S. Will, A. Leipertz, A. Fröba, P. Density, Surface tension, and kinematic viscosity of hydrofluoroethers HFE-7000, HFE-7100, HFE-7200, HFE-7300, and HFE-7500. J. Chem. Eng. Data 60 (2015) 3759-3765. 